\title{
EL USO DE ACTIVIDAD DE LATORATORIO DE BIOLOGÍA PARA LA ENSEÑANZA DE MATEMÁTICA EN LOS AÑOS INICIALES: UNA ESTRATEGIA INTERDICIPLINARIA DE ENSEÑANZA Y APRENDIZAJE
}

\author{
Jacira Helena Bridi* \\ Marilaine de Fraga Sant' Ana** \\ Marlise Geller*** \\ Juliana da Silva****
}

RESUMEN: Objetivando la interdisciplinariedad y considerándose las dificultades en el proceso de enseñanza y aprendizaje, este trabajo ha articulado una propuesta metodológica entre la Biología y la Matemática, desarrollada con dos grupos de $4^{\circ}$ año de la Enseñanza Fundamental de las redes pública y particular en Brasil, donde han sido realizadas actividades prácticas de laboratorio de Ciencias. El uso del método Allium cepa como estrategia en el estudio de fracciones, medidas y gráficos ha posibilitado la observación y el registro de datos, desarrollando igualmente el razonamiento científico y aportando el desarrollo de destrezas manipulativas. Los resultados obtenidos en esta investigación son animadores y relevantes, visto que el trabajo en laboratorio ha sido de gran provecho en el aprendizaje en Ciencias para los alumnos apuntados. Frente a los resultados, podemos observar todavía que la aplicación del test Allium cepa, a través del desarrollo de modelos matemáticos, ha desencadenado cambios significativos tanto en la postura docente cuanto en la discente.

Palabras-clave: Interdisciplinariedad; Actividades de Laboratorio; Modelo Matemático; Método Allium cepa; Enseñanza de Biología y Matemática.

\section{USE OF BIOLOGY LABORATORY ACTIVITY}

IN THE TEACHING OF MATHEMATICS IN THE EARLY YEARS:

\section{AN INTERDISCIPLINARY STRATEGY FOR TEACHING AND LEARNING}

ABSTRACT: Aiming interdisciplinary and considering the difficulties in the process of teaching and learning, this paper has articulated a methodological proposal between Biology and Mathematics, developed by two groups of $4^{\text {th }}$ year of Basic Education of public and private institutions in Brazil, where activities have been conducted using laboratory practice of Sciences. The use of Allium cepa as a strategy method in the study of fractions, measurement, and graphics has made possible the observation and recording of data, developing scientific reasoning and also providing manipulative skill development. The results obtained in this study are encouraging and relevant, given that the laboratory activity has been of great benefit in science learning for students. Compared to the results, we can still see that the application of Allium cepa test, through the development of mathematical models, has triggered significant changes in both the teaching position as the learner.

Keywords: Interdisciplinary; Laboratory Activities; Mathematical Model; Allium cepa Test; Biology and Mathematics Teaching. 
Jacira Bridi | Marilaine Sant, Ana | Marlise Geller | Juliana da Silva

\section{INTRODUCCIÓN}

La gente aplica conceptos numéricos, hace operaciones, calcula medidas y utiliza el razonamiento lógico de forma rutinaria. Éstas son destrezas que deben adquirirse ya en los primeros años escolares. Así, la Matemática en la Enseñanza Fundamental no debe verse tan sólo como un requisito previo a los estudios posteriores. Es necesario que la enseñanza de esta asignatura esté direccionada hacia la formación del ciudadano, que utiliza cada vez más conceptos matemáticos en su cotidiano. Se puede decir que Matemática y realidad son dos conjuntos disyuntos y que el modelado es un medio de hacerlas interaccionar.

El uso de modelos y de modelización en la enseñanza ha sido un tema recurrente de investigación en el área de educación científica (KAPRAS et al., 1997; Gilbert y Boulter, 2000). Esas investigaciones revelan el rol ocupado por los modelos en el aprendizaje de contenidos científicos (NERSESSIAN, 1995). En menor cantidad, algunas investigaciones se han dedicado a evaluar las formas y los resultados de actividades de modelización en la enseñanza de Ciencias (TINBERGHIN, 1994). Pero pocas investigaciones están discutiendo el tema relativamente a la presentación de modelos en libros de Ciencias (RUTHERFORD, 1995). Modelos científicos son extensamente presentados en los libros didácticos como hechos estáticos. Ello reduce la posibilidad de que se creen estrategias de enseñanza constructivistas, una vez que los libros raramente invitan a los alumnos a que construyan efectivamente, a que testen y repasen modelos (VAN DRIEL y VERLOOP, 2002).

Justi y Gilbert (2001) demuestran la importancia de modelos también para la enseñanza de Ciencias. Los autores observan que, para que los alumnos tengan una visión más amplia sobre modelos, se muestra imprescindible que sus profesores reconozcan la importancia de dichos modelos en la enseñanza y que comprendan claramente la naturaleza de modelo y de modelado.

La búsqueda de nuevas alternativas a la enseñanza indica que la Matemática ofrece un campo riquísimo a la proposición de situaciones desafiadoras en las que no se objetiva exclusivamente la fijación de contenidos, sino que se incentiva la creatividad y la originalidad paralelamente a la observación, análisis y razonamiento lógico (CARRETONI, 1986).

La formación interdisciplinaria se constituye una exigencia básica en la sociedad contemporánea. Debe tomarse como un imperativo de los más importantes de las nuevas condiciones de la producción del conocimiento científico, como también de las nuevas condiciones de ser y de estar en un mundo en red. Una formación interdisciplinaria está en sintonía con el tiempo-espacio del que forma parte, insiste en la acciones en movimiento, y sus producciones son contextualizadas (SIQUEIRA, 2003). El mismo autor comenta que trabajar la interdisciplinariedad no significa negar las especialidades y la objetividad de cada ciencia. La interdisciplinariedad debe respetar el territorio de cada campo del conocimiento, así como distinguir los puntos que los unen y los diferencian. Es ésa la 
condición necesaria para que se detecten las áreas donde se puedan entablar las conexiones posibles.

La interdisciplinariedad, como anhelo emergente de superación de la racionalidad científica positivista, aparece como entendimiento de una nueva forma de institucionalizar la producción del conocimiento en los espacios de la investigación, en la articulación de nuevos paradigmas curriculares y en la comunicación del proceso percibir las varias asignaturas; en las determinaciones del dominio de las pesquisas, en la constitución de los lenguajes compartidos, en las pluralidades de los saberes, en las posibilidades de cambios de experiencias y en los modos de realización del trabajo en equipo. Tal realización integrativo-interactiva nos permite visualizar un conjunto de acciones interconectadas de carácter totalizante y exento de cualquier visión fraccionada, superándose las actuales fronteras disciplinarias y conceptuales (SIQUEIRA y PEREIRA, 1995).

Según Botelho (2005), la perspectiva del trabajo interdisciplinario implica una trama compleja de concepciones, principios y conceptos para la realización de una trasposición didáctica adecuada y eficiente que responda tanto a los objetivos generales del proyecto interdisciplinario como a las necesidades puntuales de cada asignatura en particular, sabiéndose que ninguna disciplina aisladamente podrá dar respuestas a la complejidad de los problemas de la contemporaneidad, cuando hablar de Educación implica hablar de cultura, globalización, mercado, tecnologías de comunicación y de información.

Entre las estrategias de enseñanza consideradas constructivistas e interdisciplinarias, encuéntranse las actividades prácticas de laboratorio.

Borges $(1997,2001)$ argumenta que las actividades prácticas pueden ser fundamentales en el proceso de aprendizaje si se las planea cuidadosamente, teniendo en cuenta los objetivos pretendidos, los recursos disponibles y los conceptos previos de los estudiantes sobre el tema.

\section{EL PROCESO DE INVESTIGACIÓN}

La investigación tuvo como propósito desarrollar actividades de laboratorio de Biología con alumnos de dos grupos de $4^{\circ}$ año de la Enseñanza Fundamental, con vistas a la obtención de una base de conocimiento. No sólo estribaba en observaciones, sino en la forma interactiva entre contenidos, auxiliando la comprensión de conceptos matemáticos y brindando situaciones desafiadoras e interdisciplinarias.

El grupo 1 fue compuesto por 22 alumnos de una escuela de la red particular en el período de la mañana, y el grupo 2 fue formado por 28 alumnos de la red pública en el período de la tarde. La edad promedio para ambos grupos fue de 10 años. Se ha utilizado una muestra de 16 alumnos de cada escuela, siendo para el grupo 1, 7 niños y 9 niñas y, para el grupo 2, 6 niños y 10 niñas, pues éstos han sido los que han participado de todo el proceso de investigación. Los profesores titulares de cada grupo también han formado parte de este estudio. 


\subsection{Instrumentos de recolección de datos}

Han sido utilizados como instrumentos de recolección de datos para los alumnos: el pre y el post-test. El pre y post-test contenían las mismas cuestiones, dos abiertas sobre fracciones y una abierta sobre gráficos (Anexo 1).

La eficiencia de la metodología utilizada en la construcción del conocimiento fue evaluada a través del análisis de las respuestas, des el pre y el post-tests de los 32 alumnos, obtenidos en la institución pública y en la privada, de forma cualitativa y cuantitativa. El pre y el post-tests analizados fueron tan sólo de los alumnos que han participado de todo el trabajo, en un total de 16 alumnos de cada escuela.

La comparación de los cuestionarios fue realizada a partir de la proposición de rangos de análisis, con base en el trabajo de Da Silva e Neto (2003), para que reflejaran la comprensión del tema abordado. Las respuestas han sido analizadas, buscándose puntos en común que posibilitaran el agrupamiento.

De esta forma, el pre y el post-test realizados por los participantes han sido reunidos en:

a) CLASE 0: Sin respuesta - Respuesta del tipo "no lo sé" o en blanco;

b) CLASE 1: Respuesta Pobre - Respuestas que no indicaban comprensión por parte del alumno sobre el tema;

c) CLASE 2: Respuesta Débil-Respuestas que manifiestan cierta comprensión de los conceptos, pero de forma incompleta;

d) CLASE 3: Respuesta Satisfactoria - Respuestas que demuestran comprensión de los elementos científicos más importantes.

Para permitir una evaluación general sobre las respuestas para cada cuestión se ha atribuido un valor numérico relativo a la Clase. Sumándose los valores asignados para cada cuestión por individuo, se engendró un valor arbitrario. Ese índice arbitrario se ha atribuido a cada sujeto evaluado, variando de 0 (las tres cuestiones clase 0 , por lo tanto 3 X 0), hasta 9 (las tres cuestiones clase 3 , por lo tanto 3 X 3). Para la evaluación general, se realizó la media y la desviación patrón por grupo, siendo comparados por el test estadístico $t$-Student, donde $\mathrm{P}<0,05$ fue considerado significativo.

Las profesoras titulares de los grupos $(n=2)$, al final de los encuentros, han sido entrevistadas en cuanto a sus juicios sobre la eficiencia de las actividades realizadas.

\subsection{Actividades desarrolladas}

Inicialmente fueron realizados encuentros con las profesoras en los que la propuesta de trabajo ha sido discutida. Fue propuesto a las profesoras y a los alumnos el uso del tema cebolla - Allium cepa para abordar contenidos matemáticos tales como: fracciones, medidas y gráficos.

El test Allium cepa fue realizado de acuerdo con el protocolo descrito por Babich et al. (1997), con algunas modificaciones y adaptado a la enseñanza 
de Matemática en los años iniciales. Las cebollas utilizadas en el test deben ser de tamaño pequeño, uniforme, de mismo origen, no germinadas y sanas. Los bulbos fueron puestos para germinar sobre recipientes apropiados, con la parte inferior zambullida en solución test. Cada prueba completa consistió de tres bulbos, siendo cada cual zambullido en solución distinta. A los diferentes grupos de alumnos les ha tocado decidir en qué soluciones se expondrían los bulbos: (A) Agua; (B) Café + Agua (dos concentraciones distintas). Tras algunos días de germinación, aproximadamente una semana, fueron hechas tomas de datos en cuanto al desarrollo de las raíces de acuerdo con la solución. Con los datos, los alumnos han sido estimulados a crear modelos matemáticos para el crecimiento de las raíces. Las actividades fueron realizadas alrededor de cuatro semanas. Con los alumnos, pesquisa bibliográfica, discusiones y actividad de laboratorio han sido desarrolladas.

El test Allium cepa fue elegido debido al hecho de que la cebolla se la emplea ampliamente para estudios de toxicidad y genotoxicidad, tiene bajo coste, es de fácil obtención y manipulación. Este organismo se usa normalmente para pruebas de contaminación de aguas por efluentes específicos o contaminación en general. Distintos autores (FISKEJÖ, 1993; GOMEZ-ARROYO et al., 1986; MA et al., 1995; COTELLE et al., 1999; MARCANO et al., 2004) defienden la metodología Allium cepa como una de las mejores para el monitormiento ambiental, usándose diferentes parámetros, como crecimiento de las raíces y errores en la división celular para obtenerse informaciones tanto toxicológicas cuanto mutagénicas. De esta forma, por dicha amplia difusión de la metodología, el test Allium cepa comienza a recomendarse como herramienta de enseñanza. (BABICH et al., 1997).

Han sido realizados igualmente algunas discusiones referentes a los contenidos de fracciones y gráficos, así como otros sobre medidas del tipo: ¿Sabe usted utilizar adecuadamente la regla para medir los objetos? ¿ Sabe usted qué significa 1/2? Para usted, ¿qué quiere decir fracciones? Al mirar una hoja de revista o periódico encontramos gráficos. ¿Sabe usted para qué sirven?

Gran parte de las respuestas (incluso orales) fue que todavía no han trabajado esos contenidos. Algunos, sin embargo, han contestado que los gráficos que ven en revistas y periódicos sirven para "explicar algo de la tal noticia”. El pre-test fue aplicado enseguida.

Aun en el primer encuentro se ha hecho la propuesta de una investigación en internet sobre la cebolla. La investigación tuvo como objetivo encontrar el nombre científico de la cebolla, su uso y alguna curiosidad. También se ha pedido para que los alumnos crearan tiras cuyo personaje principal fuera la cebolla. Dichas actividades fueron utilizadas como una estrategia de acercamiento al grupo. Los trabajos deberían entregarse por ocasión del segundo encuentro.

- Relativamente al pre-test, que contenía dos cuestiones abiertas, se puede observar el resultado en la Tabla 1 para el Grupo 1 y en la Tabla 2 para el Grupo 2. 
Tabla 1 - Categorización de las respuestas de los individuos en cuanto a su comprensión sobre los temas antes y después de las discusiones y actividades (pre-test y post-test) para el Grupo 1.

\begin{tabular}{|c|c|c|c|c|}
\hline CUESTIONES & \multicolumn{4}{|c|}{ NÚMERO TOTAL DE ALUMNOS / CLASE DE RESPUETAS } \\
\hline $\begin{array}{c}\text { Pre-Test } \\
\text { 1) En el estudio de las fracciones, ¿qué entiende } \\
\text { usted por }\end{array}$ & $\begin{array}{c}\text { Satisfactoria } \\
\text { (3) }\end{array}$ & $\begin{array}{l}\text { Débil } \\
\text { (2) }\end{array}$ & $\begin{array}{l}\text { Pobre } \\
\text { (1) }\end{array}$ & $\begin{array}{c}\text { Sin respuesta } \\
\text { (0) }\end{array}$ \\
\hline 1. a) conjunto discreto? & 0 & 0 & 0 & $16(100 \%)$ \\
\hline 1.b) conjunto continuo? & 0 & 0 & 0 & $16(100 \%)$ \\
\hline 2) ¿Qué entiende usted al observar este gráfico?* & 0 & $2(12,5 \%)$ & $12(75 \%)$ & $2(12,5 \%)$ \\
\hline $\begin{array}{c}\text { Post-Test } \\
\text { 1) En el estudio de las fracciones, ¿qué entiede } \\
\text { usted por }\end{array}$ & $\begin{array}{c}\text { Satisfactoria } \\
\text { (3) }\end{array}$ & $\begin{array}{l}\text { Débil } \\
\text { (2) }\end{array}$ & $\begin{array}{l}\text { Pobre } \\
\text { (1) }\end{array}$ & $\begin{array}{c}\text { Sin respuesta } \\
\text { (0) }\end{array}$ \\
\hline 1. a) conjunto discreto? & $2(12,5 \%)$ & $10(62,5 \%)$ & $1(6,2 \%)$ & $3(18,8 \%)$ \\
\hline 1.b) conjunto continuo? & $3(18,8 \%)$ & $8(50 \%)$ & $5(31,2 \%)$ & 0 \\
\hline 2) ¿Qué entiende usted al observar este gráfico?* & $1(6,2 \%)$ & $4(25 \%)$ & $8(50 \%)$ & $3(18,8 \%)$ \\
\hline
\end{tabular}

*La figura del test está en el Anexo 1.

Tabla 2 - Categorización de las respuestas de los individuos en cuanto a su comprensión sobre los temas antes y después de las discusiones y actividades (pre-test y post-test) para el Grupo 2.

CUESTIONES

Pre-Test

1) En el estudio de las fracciones, ¿qué entiende usted por

1. a) conjunto discreto?

1.b) conjunto continuo?

2) ¿Qué entiende usted al observar este gráfico?* Post-Test

1) En el estudio de las fracciones, ¿qué entiede usted por

1. a) conjunto discreto?

1.b) conjunto continuo?

2) ¿Qué entiende usted al observar este gráfico?*

NÚMERO TOTAL DE ALUMNOS / CLASE DE RESPUETAS

\begin{tabular}{|c|c|c|c|}
\hline $\begin{array}{c}\text { Satisfactoria } \\
\text { (3) }\end{array}$ & $\begin{array}{l}\text { Débil } \\
\text { (2) }\end{array}$ & $\begin{array}{l}\text { Pobre } \\
\text { (1) }\end{array}$ & $\begin{array}{c}\text { Sin respuesta } \\
\text { (0) }\end{array}$ \\
\hline $\begin{array}{l}0 \\
0 \\
0\end{array}$ & $\begin{array}{c}0 \\
0 \\
1(6,2 \%)\end{array}$ & $\begin{array}{c}0 \\
0 \\
7(43,8 \%)\end{array}$ & $\begin{array}{c}16(100 \%) \\
16(100 \%) \\
8(50 \%)\end{array}$ \\
\hline $\begin{array}{c}\text { Satisfactoria } \\
\text { (3) }\end{array}$ & $\begin{array}{l}\text { Débil } \\
\text { (2) }\end{array}$ & $\begin{array}{c}\text { Pobre } \\
\text { (1) }\end{array}$ & $\begin{array}{c}\text { Sin respuesta } \\
\text { (0) }\end{array}$ \\
\hline $\begin{array}{c}4(25 \%) \\
5(31,3 \%) \\
1(6,2 \%)\end{array}$ & $\begin{array}{c}11(68,8 \%) \\
9(56,3 \%) \\
11(68,8 \%)\end{array}$ & $\begin{array}{c}0 \\
1(6,2 \%) \\
4(25 \%)\end{array}$ & $\begin{array}{c}1(6,2 \%) \\
1(6,2 \%) \\
0\end{array}$ \\
\hline
\end{tabular}

\footnotetext{
*La figura del test está en el Anexo 1.
}

En lo que toca a la cuestión 2, señalamos algunas respuestas que ilustran lo observado:

- Alumno JF: "Que este gráfico representa el aprendizaje del alumno". (CLASE 1).

- Alumno GP: "Yo noté que inglés está igual al portugués. Carol está mala en Matemática". (CLASE 2).

Relativamente a las investigaciones desarrolladas por los alumnos, los resultados fueron diversificados, destacándose los contenidos de Ciencias, Portugués, Geografía e Historia. En las actividades que abarcaban la elaboración de tiras, la creatividad ha sido sorprendente. Han surgido personajes interesantes y también ha sido posible observar el fuerte enfoque hacia la importancia de la alimentación y las vitaminas. Fueron creadas historias como "La Graduación de Allium cepa” (Figura 1) y e "El Juicio" (Figura 2). La discusión reconstructiva 
se hizo a través del diálogo oral y escrito. Así, otro expediente de la pesquisa está en el ejercicio de la escrita, pues, por su medio, se construye la capacidad de argumentación. Es necesario que se incentive a los alumnos a que construyan un texto, teniendo alguna elaboración propia. De otra parte, tal proceso de lectura y de escrita favorece igualmente el desarrollo de otra característica esencial de la pesquisa que es la socialización del argumento (GALIAZZI et al., 2001).

Figura 1: "La Graduación de Allium cepa".

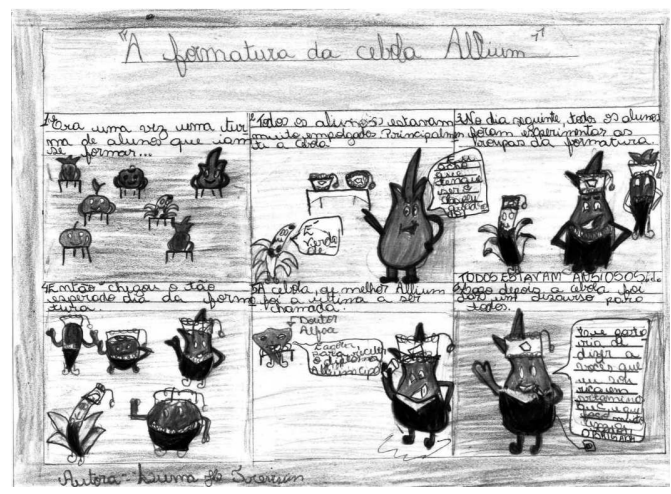

Figura 2: "El Juicio".

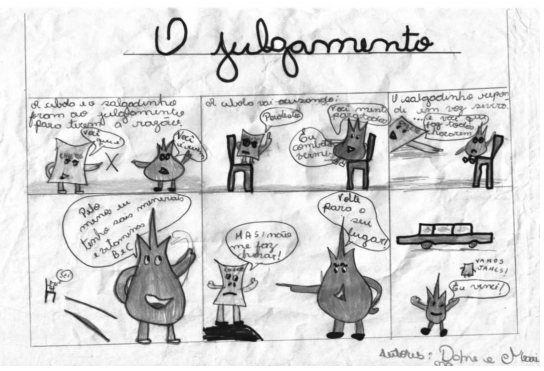

En el laboratorio, los alumnos se reunieron en grupos y dieron inicio al test Allium cepa. Tal procedimiento fue realizado en las dos escuelas. Cada grupo formado ha elegido un dibujo para identificarlos, como por ejemplo: corazón, estrella, flor, etc. La metodología de laboratorio utilizada fue el test Allium cepa (cebolla), conforme descrito a seguir:

Los materiales utilizados fueron: 15 bulbos de cebolla; 15 envases pet; 30 pinchitos; agua; solución concentrada de café (agua + café).

Los siguientes procedimientos fueron seguidos: cada grupo recibió 3 bulbos de cebolla y 3 envases pet para efectuar el test. A cada grupo le tocó el cambio de agua 2 veces a la semana. Cada grupo elaboró una tabla donde se tomarían los datos de crecimiento de las raíces para posterior elaboración del informe, discusión y creación de los modelos matemáticos. 
Durante la actividad de laboratorio, se ha explicado a los alumnos que los 15 bulbos de cebolla formaban un conjunto que es un ejemplo de conjunto discreto (conjunto de elementos iguales, de misma especie).

Se han hecho algunas preguntas del tipo: ¿Cuál el total de cebollas? "15" - respuesta de los alumnos. ¿Cuántas cebollas le ha tocado a cada grupo? “3” - respuesta de los alumnos. ¿Tres cebollas de un total de cuántas? "15” - respuesta de los alumnos. ¿Qué se puede concluir? Que a cada grupo le tocarán 3 de un total de 15 cebollas, o sea: 3/15 del todo (conjunto de cebollas). En este caso hay la fracción 3/15, donde el valor 3 es el numerador y 15 es el denominador - respuesta discutida entre profesores y alumnos.

Luego de recibir los tres recipientes (envases pet), se solicitó a cada grupo los que numeraran de 1 a 3 y que fueran llamados P1, P2 y P3. Todavía les fue pedido que identificara a qué grupo pertenecía cada material a través del dibujo anteriormente elegido por ellos (estrella, flor, corazón, etc.), facilitando de este modo la identificación para el procedimiento de las actividades en los encuentros posteriores.

En P1 fue colocado tan sólo agua. Se solicitó que todos los grupos pusieran la misma cantidad total de líquido: se discutió entonces que el total del líquido (4 vasos) formaría el todo, que, en este caso, fue un ejemplo de conjunto continuo. Mientras el agua se colocaba junto a los grupos del P1, se cuestionaba también que 4 de 4 vasos son de agua, lo que formaba la fracción 4 de 4 , $4 / 4=1$ (todo).

En P2 se ha mezclado 3 vasos de agua y 1 de solución de café, en que se discutió que 3 de 4 son de agua y que forma la fracción $3 / 4$, y que 1 de 4 es de solución de café, formando la fracción $1 / 4$. Se subrayó que, si sumados $3 / 4+1 / 4=$ $4 / 4=1$ (todo).

En P3 se han mezclado 2 vasos de agua y 2 de solución de café, en que se observó que 2 de 4 de agua forman la fracción $2 / 4$ y que 2 de 4 de agua con café forman igualmente la fracción $2 / 4$, así, sumándose $2 / 4+2 / 4=4 / 4=1$ (todo).

Con esta etapa del test ha sido posible discutir las partes iguales de un todo (considerándose el conjunto de cebollas y el conjunto de agua) y citar también como ejemplos de conjunto DISCRETO las cebollas y el agua como conjunto CONTINUO. A estas partes iguales de un todo fue comentado que llamamos de fracciones y que le damos un nombre a cada número: numerador y denominador.

Los alumnos discutían y participaban activamente, observando que, a partir de esta actividad, han podido aprender sobre fracciones. Durante la semana, la profesora titular ha aprovechado la idea para trabajar más con las fracciones, así como con otros cálculos y también otras asignaturas. Fue elaborado un pequeño informe por ítemes del estudio de la cebolla.

Cada grupo ha puesto un bulbo zambullido en cada solución con el soporte de dos pinchitos para que, de esta forma, no se hundieran o se volcaran. La parte que quedó en contacto con la solución fue cuidadosamente limpia. 
Les fue solicitado que hicieran la medición de las raíces a cada dos días, en los mismos días en que las soluciones fueran cambiadas, siendo tales medidas apuntadas en la tabla elaborada por ellos.

$\mathrm{Al}$ inicio del tercer encuentro, fueron retomadas las actividades de los encuentros anteriores y fue organizada la tabla de datos del crecimiento de las raíces (Figura 3 - A e B).

Comentarios sobre los apuntes y observaciones fueron realizados. Las discusiones abarcaron principalmente la observación de que el crecimiento de las raíces fue mejor en P1. Para ellos quedó claro que era debido a que éste contuviera solamente agua y que en los demás el crecimiento quedó más lento, siendo que en P2 creció un poco más que en P3, correspondiendo a las concentraciones de café. En este punto, han surgido discusiones sobre la toxicidad del café y sus efectos biológicos.

Figura 3 (A y B): Tablas con los datos obtenidos de las medidas de las raíces por el grupo Flor (A) y por el grupo Estrella (B).

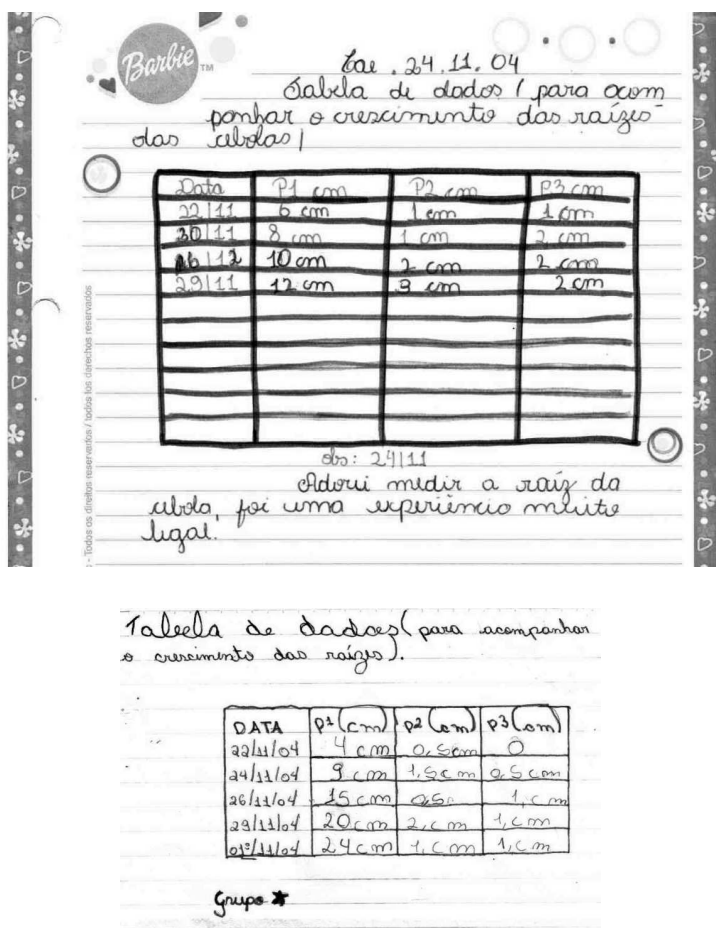

Tras la observación, el análisis y la discusión de los datos recolectados y tabulados, cada grupo elaboró un gráfico de barras en que fueron usados como coordinadas el crecimiento $\mathbf{c m}$ (eje y) y cada recipiente (eje x) (Figura 4 - A y B). 
Figura 4 ( $\mathrm{A}$ y $\mathrm{B}$ ): Gráfico elaborado por el grupo Flor (A) y por el grupo Estrella (B).
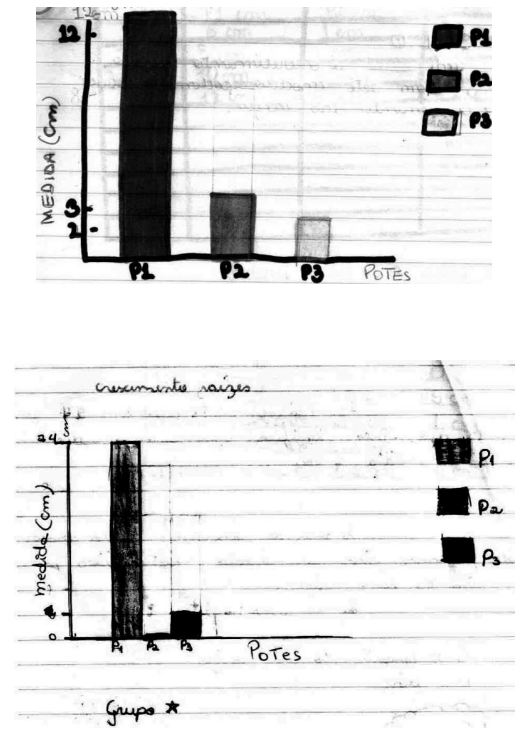

Tras la creación de los gráficos, fueron hechas preguntas orales del tipo: “Qué podemos observar en cuanto al tamaño de cada columna del gráfico?” Los alumnos contestaron que cada columna indica el crecimiento de las raíces en aquel determinado recipiente. Comentaron que, al observar el gráfico, quedó clara la diferencia entre el crecimiento de cada recipiente. Así, han logrado una visión comparativa de los datos recolectados durante los días de "cultivo".

Con los mismos datos recolectados y tabulados, fueron calculados los promedios de crecimiento para cada grupo. Se decidió calcular solamente el promedio de P1, pues en éste el crecimiento fue superior. Los grupos fueron orientados sobre cómo proceder para el cálculo del promedio de crecimiento diario de las raíces: si el crecimiento de las raíces fue acompañado por 12 días, entonces cada grupo debería utilizar el total del crecimiento, el cual es la medida del último día de tabulación de datos y divido por el número de días, en este caso, 12. Cada grupo realizó dicho cálculo, obteniendo así el promedio de crecimiento diario de las raíces de cebolla para la situación. De este modo, por ejemplo, para el grupo FLOR, en los 12 días, las raíces crecieron $12 \mathrm{~cm}$, entonces 12:12 $=1$. Así, el promedio para el grupo FLOR fue de $1 \mathbf{c m}$ al día. Ya para el grupo Estrella, en los 12 días, las raíces crecieron $24 \mathrm{~cm}$. Entonces 24:12=2. De este modo, el promedio para el grupo Estrella fue de $2 \mathrm{~cm}$.

A partir de esos datos, cada grupo ha analizado las informaciones de la tabla, del gráfico y de las medidas que poseían. Además, han creado un modelo matemático del crecimiento de las raíces del período observado. 
Cada grupo pudo entonces discutir los valores encontrados, y luego se ha convenido que, para la creación del modelo matemático, se utilizarían las siguientes notaciones: “c” para la longitud de las raíces y "d" para día, donde "c" y "d" son las variables, y el promedio calculado por cada grupo es constante. Así, los distintos grupos obtuvieron un modelo matemático como se puede observar en los ejemplos a seguir:
(a) grupo Flor:
$c=1 . d$
(b) grupo Estrella:
$c=2 . d$

Tras la obtención de estos modelos, cada grupo tuvo como desafío calcular el crecimiento de las raíces para $\mathbf{n}$ días, esto es, prever el crecimiento de las raíces por más días que los acompañados y observados. Los alumnos se mostraron satisfechos al poder crear un modelo (fórmula) para esa situación. Cada grupo realizó cálculos para cerca de 30, 60 y 90 días. El Grupo Estrella, por ejemplo, logró concluir que, si la raiz crece $2 \mathrm{~cm}$ al día, utilizando el modelo que construyeron, al cabo de 60 días habría crecido $120 \mathrm{~cm}$.

Según Biembengut (2003), no importa el caso, la resolución de un problema, en general cuando cuantificado, requiere una formulación matemática detallada. En esa perspectiva, a un conjunto de símbolos y relaciones matemáticas que busca traducir de algún modo un fenómeno en cuestión o problema de situación real se lo denomina "modelo matemático". Con los datos, tras las discusiones, fue posible la elaboración del modelo matemático para el crecimiento de las raíces de la cebolla en esta situación por los grupos. El modelo matemático en este trabajo se puede considerar Educacional Aplicativo por ser utilizado para fines educacionales, y Dinámico, por explicar un fenómeno que ha evolucionado con el tiempo, pues, a través de él ha sido posible simular variaciones de estadios del fenómeno del crecimiento de las raíces de cebolla.

\subsection{El envolvimiento de los professores}

Los profesores titulares han aprovechado la idea y han profundizado otras cuestiones como: (a) Región de mayor producción de la cebolla; (b) Textos complementarios; (c) Cálculos acerca del precio de la cebolla en el mercado, en la CEASA, en el campo; (d) La utilidad de la cebolla para la salud; (e) Otros temas.

Entre un encuentro y otro, los grupos quedaron responsables por el "cuidado" del cultivo de las cebollas. Los datos (medida de las raíces) fueron tomados en cinco distintas fechas: $1^{\text {er }}$ día, $3^{\text {er }}$ día, $5^{\circ}$ día, $8^{\circ}$ día y $10^{\circ}$ día (algunos grupos, 12 días). Cada grupo hizo una tabla con las medidas del crecimiento de las raíces, identificando cada recipiente con su debido número: P1 = representa- 
ba la solución A (solamente agua); P2 = representaba la solución B (agua + café, 3:1 v/v); P3 = representaba la solución B y distinta concentración (agua + café, $2: 2 \mathrm{v} / \mathrm{v}$ ). También en el período entre los encuentros, las profesoras han trabajado con las fracciones a partir de las actividades levantadas durante la aplicación del test Allium cepa. Fue posible desarrollar algunas actividades de suma de fracciones, realizar la lectura de fracciones, reconocer las nomenclaturas de cada número que compone la fracción, entre otros cálculos planteados por esta metodología.

\subsection{Analizando la experiencia}

Al finalizar las actividades, se aplicó el post-test, el cual presentaba las mismas cuestiones del pre-test. Junto al post-test, los alumnos dejaron algunos apuntes sobre la realización del test Allium cepa., si les había gustado, si había sido provechoso, si había sido una experiencia interesante, entre otras observaciones.

Fue posible constatar que la ida al laboratorio les dejó a los alumnos fascinados. Todo les llamó su atención. La rutina de la sala de clase fue rota, y cada encuentro era esperado con gran emoción y euforia. Los alumnos se han mantenido constantemente desafiados por la metodología, pues ésta le exigió a cada grupo un cuidado y una observación la investigación. En el grupo 2 sucedió algo desagradable, pero a la vez curioso e interesante. En el período de la mañana, un alumno resolvió cortar las raíces de las cebollas de uno de los grupos de la pesquisa. Tal hecho chocó a los alumnos, que informaron lo sucedido. La preocupación de no poder más acompañar el crecimiento de las raíces quedó visible, y con ello demostraron el real interés en desarrollar el trabajo planteado. Tanto es así que, al tabular los datos y crear el gráfico (puesto que ya habían realizado la toma de algunas de las medidas) dejaron el registro de su indignación en lo que toca a la actitud que, según el grupo, los ha perjudicado.

Se puede subrayar el comentario del alumno AG:

"O Recipiente 1 fue el que más creció, pues tenía tan sólo agua. Algunos días después, los alumnos del período inverso cortaron las raíces. OBS: las raíces fueron cortadas, perjudicando nuestro trabajo."

Comparándose pre y post-test para los dos grupos y observándose las tablas 1 y 2 , se puede verificar que, tras las actividades, hubo una mejoría en las respuestas, donde se puede, incluso, constatar algunas respuestas del tipo satisfactoria.

Para la cuestión 1.b, de los 16 alumnos del grupo 1: 18,8\% presentaron respuestas del tipo no lo sé o en blanco; $6,2 \%$ contestaron que es un conjunto que se puede separar; $62,5 \%$ que es un conjunto de mismos objetos; $12,5 \%$ que es un conjunto de objetos iguales, dando, incluso, ejemplos. Para los 16 alumnos del grupo 2 , fueron observados $6,2 \%$ de las respuestas del tipo en blan- 
co; $68,6 \%$ que es un conjunto de mismos objetos; $25 \%$ que es un conjunto de objetos iguales, dando, incluso, ejemplos. El alumno HD da un ejemplo de conjunto discreto: "15 recipientes y 15 cebollas"; JK ejemplifica con "15 mesas, 15 sillas". BD comenta que: "Un conjunto discreto son varias cosas y objetos parecidos.” El alumno GC ejemplifica: "Un grupo (conjunto) de globos, recipientes, cebollas, tomates, etc.”. Todos los ejemplos corresponden a la CLASE 3.

Para la cuestión 1.b, de los 16 alumnos del grupo 1, 31,2\% citan como respuesta conjunto del agua; $50 \%$ que es un conjunto de los líquidos; $18,8 \%$ que es un conjunto de los líquidos (que no se reparte siempre de modo igual), dando incluso ejemplos. De los 16 alumnos del grupo 2, 6,2 han dejado la respuesta en blanco; 6,2\% apuntan el conjunto del agua; $56,3 \%$ que es un conjunto de los líquidos; $31,3 \%$ que es un conjunto de los líquidos (que no se reparte siempre de modo igual), dando incluso ejemplos. El alumno PG da un ejemplo de conjunto continuo: "Es un conjunto donde se reparte un objeto en varios trozos." El alumno BR comenta: "El continuo es del tipo que usted pone el agua en el recipiente, pero no logra sacar la misma cantidad." La alumna GC ejemplifica: "El conjunto continuo es tan sólo una cosa que podemos repartir en varios trozos y tamaños.". Todos los ejemplos corresponden a la CLASE 3. Se ha observado que, en la cuestión 1.a y 1.b, los ejemplos han quedado en torno del tema de la actividad de laboratorio (algo del tipo lúdico para el concreto).

En la cuestión 2, para los 16 alumnos del grupo 1 se observa que $18,8 \%$ de las respuestas son en blanco; $50 \%$ contestaron que eran los apuntes de los alumnos; 25\% que era el placer de cada alumno por la materia; 6,2\% que representaba en que asignatura el alumno estaba mejor, dando ejemplo. El alumno JB da el siguiente ejemplo: "Joana es mejor en Portugués, 90; Andrés, en Matemática, 50, e Inglés, 40.” LT comenta: "La peor es Ana en Portugués, y la mejor es Joana, en Portugués. En Inglés, todos son iguales. En Matemática, todos son distintos.”. Todos los ejemplos corresponden a la CLASE 3.

Se observa que, tras el trabajo, los alumnos tuvieron otra interpretación de las cuestiones, como se puede constatar al compararse las respuestas del alumno GC para el pre-test relativamente al post- test:

Pre-test: "Yo entendí que cada cual fue mejor que el otro, pero cada cual tiene su punto fuerte y su punto débil.." (CLASE 1).

Post-test: "Yo entiendo que uno se haya salido mejor que el otro. El gráfico ha ayudado a percibir el grado de cada cual. Joana se salió mejor en Matemática, y cada cual tiene su punto débil y su punto fuerte." (CLASE 3).

De modo general, la diferencia entre pre y post-test queda más evidente cuando se observan las Figuras 5 y 6 . Se constata que todos los estudiantes de ambas instituciones de enseñanza investigadas presentaron una "evolución conceptual" tras las actividades. 
Figura 5 - Índice Arbitrario generado para cada alumno del grupo 1, considerando las clases de respuestas para las tres cuestiones.

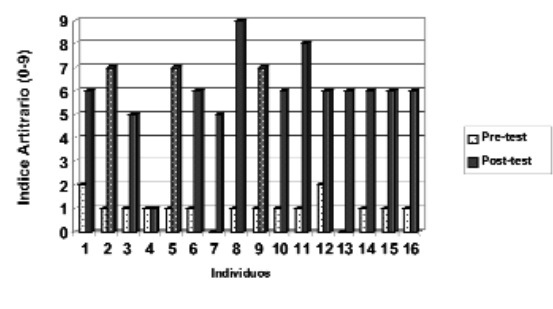

Figura 6 - Índice Arbitrario generado para cada alumno del grupo 2, considerando las clases de respuestas para las tres cuestiones.

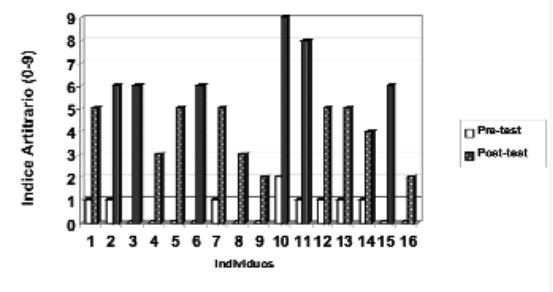

Tal se confirma al averiguar la diferencia a través del test $t$-Student entre los promedios del índice arbitrario para las cuestiones 1.a y 1.b (Figuras 7 y 8). Se puede observar que, tanto para el grupo 1 como para el grupo 2, hubo un aumento significativo en los resultados del post-test relativamente al pre-test. Ello nos permite decir que la metodología utilizada fue eficiente para la enseñanza de fracciones. Ya en la cuestión 2, para el Grupo 2, se observa una mejoría más grande de lo que sucedió con el Grupo 1, tal vez debido a que las respuestas hayan quedado en el ámbito del análisis visual de la figura del gráfico (ver Anexo 1). No se ha observado riqueza en las respuestas, quedando así sin mucha diferencia el promedio del índice arbitrario del pre y del post-test. La metodología para esta escuela, y en lo que concierne a la interpretación de los gráficos, no tuvo el mismo éxito, quizá debido a que los alumnos observaran el gráfico del post-test y no hubieran logrado establecer relación de éste con el que ellos construyeron, por tener el uno el enfoque de apuntes y el otro el del crecimiento de raíces. Pese a que la metodología no haya sido tan eficiente en ambos grupos en la discusión de gráfico, se observa igualmente una mejoría, pero la significación de "aumento de conocimiento" queda principalmente relacionada a la cuestión 1 , concerniente a fracciones. De este modo, se constata que la actividad práctica de Biología en la condición en que fue realizada, test Allium cepa, ha presentado 
una influencia positiva en la enseñanza de Matemática, principalmente en lo que toca a la enseñanza de fracciones.

Figura 7: Promedio y desvío patrón de los índices arbitrarios para el Grupo 1 por cuestión y de forma general de los tests. ${ }^{*} \mathrm{P}<0,05 \mathrm{e}{ }^{* *} \mathrm{P}<0,001 /$-Student.

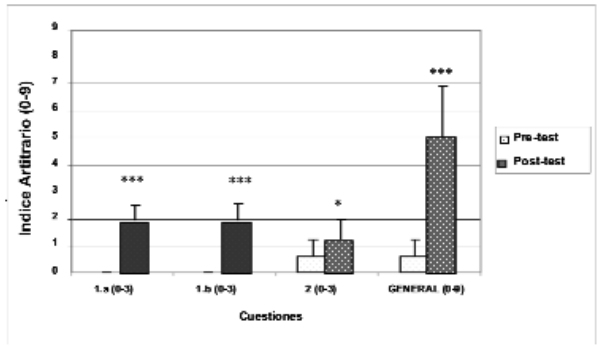

Figura 8: Promedio y desvío patrón de los índices arbitrarios para el Grupo 2, por cuestión 2 por cuestión y de forma general de los tests ${ }^{* *} \mathrm{P}<0,001 / t$-Student

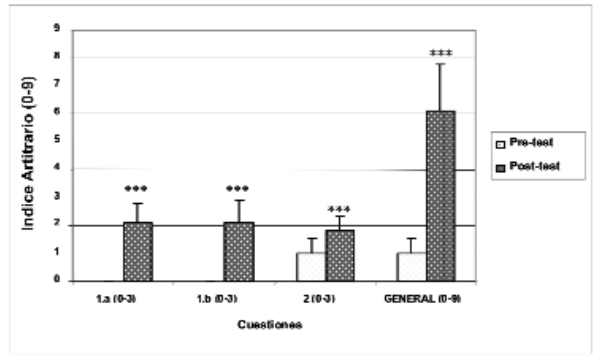

La eficiencia de la metodología queda más evidente cuando se observan los relatos en las entrevistas de las profesoras titulares. La Profesora del Grupo 1, en su entrevista, comenta:

Observé que hubo modificaciones relativamente a la motivación del grupo, pues todos han participado con más interés de las actividades propuestas. El grupo ha demostrado interés en dar continuidad a este tipo de metodología, pues consideró las actividades más interesantes, agradables y placenteras. También he observado que hubo crecimiento en relación al aprendizaje de Matemática. He observado mucha diferencia en el aprendizaje de fracciones y gráficos relativamente a grupos de años anteriores. Creo que eso sea debido a que la actividad haya sido más real para los alumnos. Yo utilizaría la metodología y también la recomendaría, pues la referida metodogía les brinda una construcción gradual del conocimiento a través de la observación práctica. 
De forma similar, la Profesora del Grupo 2 observa la eficacia de la metodología Allium cepa:

\begin{abstract}
El grupo 2, en el desarrollo del test Allium cepa, se mantuvo motivado para cuestionar, participar y observar las actividades con alegría y curiosidad. El grupo siempre ha trabajado bien y en equipos. La metodología utilizada ha exigido momentos de observación y, consecuentemente, de razonamiento crítico para elaborar las actividades posteriores. El grupo solicitó la continuidad del test. En lo que toca a la Matemática, las actividades desarrolladas han desencadenado el gusto por la elaboración de gráficos y el buen entendimiento de las fracciones. Anteriormente el estudio de las fracciones era introducido de manera "primitiva", o sea, solamente con actividades escritas y no concretas, que son para los alumnos actividades significativas que garantizan un aprendizaje eficaz. Utilizaría y recomendaría la metodología, pues está de acuerdo con las observaciones hechas en todos los momentos. Han llevado el grupo a participar, aprender, criticar y a dejarlos todavía más curiosos, esperando por las actividades siguientes.
\end{abstract}

En pesquisa realizada por Kerr, en 1963 (HODSON, 1990), época de gran difusión de las actividades experimentales en las escuelas de todo el mundo, los profesores asignaron diez motivos para la realización de actividades experimentales en las escuelas. Esos motivos fueron observados en nuestro estudio y encuéntranse repetidamente en pesquisas más recientes (HODSON, 1994), que son: (a) estimular la cuidada observación y el registro meticuloso de los datos; (b) promocionar métodos de razonamiento científico sencillo y de sentido común; (c) desarrollar destrezas de manipulación; (d) entrenarse en resolución de problemas; (e) adaptar las exigencias de las escuelas; (f) aclarar la teoría y promocionar su comprensión; ( $g$ ) averiguar hechos y principios estudiados anteriormente; (h) vivir el proceso de encontrar hechos por medio de la investigación; (i) motivar y mantener el interés en la materia; (j) hacer los fenómenos más reales por medio de la experiencia.

Así se puede igualmente subrayar que las principales actividades expresas en la Ciencia son: cumular información por medio de la observación; organizar esa información y buscar regularidades; preguntar por qué ellas aparecen y comunicar los hallazgos a los demás. O sea, todo empieza con la observación. Y esto es reforzado por los autores Galiazzi y colaboradores (2001) al afirmar: "Cuando la observación se realiza bajo control cuidadoso, es dignificada por un nombre especial - una secuencia controlada de observaciones es llamada de experiencia - toda ciencia está construida sobre resultados experimentales."

Se puede, de esta forma, señalar la necesidad de estímulo a los profesores en distintas áreas a que utilicen actividades prácticas de forma interdisciplinaria. Para tanto, la formación de profesores se vuelve esencial. De este modo, se observa que los profesores de Ciencias son doblemente afectados. Según Fourez (2003), inicialmente los profesores tienen que encontrar soluciones frente a la crisis de las escuelas, en donde perdieron poder y consideración relativamente a su profesión, y deben buscar siempre la actualización. La formación de 
los licenciados estuvo más centrada sobre el proyecto de hacerlos técnicos de ciencias que volverlos educadores. Sus estudios no están muy preocupados en introducirlos en la práctica tecnológica o en la manera como las ciencias y tecnologías se favorecen, tampoco en los intentos interdisciplinarios. Ellos confunden frecuentemente la tecnología y la aplicación de las ciencias o la aplicación de un sistema experimental. En lo que atañe a la interdisciplinariedad, raramente son enseñados a cómo hacer intervenir para resolver una situación problemática en las asignaturas pertinentes, sean ellas de ciencias naturales o humanas.

Aún conforme el autor, cuando se está más interesado por la alfabetización científica o por la formación en los métodos que por la cumulación de resultados, rápidamente se es llevado a cuestionar sobre la manera de formar profesoras hacia destrezas bastantes generales, tales como: (a) saber construir una representación clara (un "modelo") de una situación concreta; (b) saber utilizar los expertos; (c) saber cruzar, para comprender una situación, conocimientos estandarizados de las ciencias y de los abordajes singulares de usuarios; (d) saber cuánto vale la pena profundizar una cuestión y cuándo es mejor contentarse - al menos provisionalmente - con una representación más sencilla; (e) saber evaluar el nivel de rigor con el que conviene abordar una determinada situación; saber el buen uso de los lenguajes y de los saberes estandarizados; ( $f$ ) saber utilizar los saberes establecidos para aclarar una decisión o un debate; (g) saber testar la representación que se tiene de una situación, confrontándola con la experiencia en cuanto a los modelos teóricos, etc.

\section{CONCLUSIONES}

A lo largo de las actividades desarrolladas, los alumnos fueron a los pocos adquiriendo confianza y creyendo en la eficacia de la metodología. Han quedado fascinados con la ida al laboratorio y han participado activamente de todas las etapas.

Los resultados obtenidos en esta investigación son animadores y relevantes, visto que el trabajo en laboratorio ha sido de gran provecho en el aprendizaje en Ciencias para los alumnos apuntados. Les ha motivado el contenido. Esto refuerza los datos de Borges et al. (2001), donde se concluye que las actividades investigativas se pueden implementar independientemente de nuevas tecnologías. En sala de clase, los profesores pueden utilizar materiales convencionales para crear una situación de aprendizaje, teniendo como base un proceso de investigación. Ese proceso se constituye una acción innovadora, no pudiendo, de este modo, anticipar percepciones y resultados, buscando siempre desafiar a los estudiantes en la construcción de sus propios conocimientos. De la observación y análisis de los datos obtenidos, se puede inferir que la utilización de la práctica de laboratorio de Biología, el test Allium cepa, ha mostrado su eficacia en el 
desarrollo cognitivo y, consecuentemente, en la evolución conceptual de los estudiantes en lo que concierne a la Matemática, pues ha posibilitado la observación (reconocimiento de la situación-problema), registro de datos. Planteó el método de razonamiento científico sencillo y de sentido común. Brindó el desarrollo de destrezas de manipulación. Motivó y mantuvo el interés por la materia y tornó el fenómeno más real por medio de la experiencia (interpretación de la solución).

Se puede observar que el uso de esta metodología en conjunto con las clases teóricas ha permitido que los alumnos tengan una visión crítica acerca de los temas en cuestión, puesto que cada alumno puede, a lo largo de las actividades, cuestionar, hacer sugerencias, teniendo, de este modo, la oportunidad de construir su aprendizaje propio. El alumno ha sido parte activa en el desarrollo de esta metodología. Desde la introducción de las actividades, la participación fue de fundamental importancia. En el final, los alumnos mismos han podido elaborar sus conclusiones sobre cada etapa de las actividades.

Así, se constata que el profesor debe mantener su propósito de brindarles a los alumnos la experiencia del descubrimiento, permitiendo comprender el significado de las actividades desarrolladas, poniéndose como mediador, valorando e incentivando la búsqueda de respuestas por medio de sus sugerencias y cuestiones. La actitud del profesor durante todo el proceso se vuelve de fundamental importancia. Al profesor le cabe el papel primordial de incentivar y orientar la conducción del proceso de enseñanza y aprendizaje a través de metodologías como la que fue utilizada. Actividades prácticas pueden servir como impulsoras del aprendizaje significativo por estimularles a los alumnos con problemas de la vida real. Según Rogers (1971), es uno de los modos más eficaces de aprendizaje. El alumno ha constatado a lo largo del proceso lo mucho que ha sido eficaz y placentero su aprendizaje.

De esta suerte, recomendamos la utilización del test Allium cepa como una herramienta para la enseñanza de Matemática en los años iniciales, así como en los demás años y asignaturas debido a que éste es de fácil adaptación a los contenidos y a que tiene un bajo coste por la utilización de materiales alternativos.

Se sugiere que sean realizados talleres con los profesores en las escuelas para que puedan tener la oportunidad de testar dicha metodología, así como otras alternativas para trabajar los contenidos de Matematica de forma interdisciplinaria. 


\section{NOTA}

${ }^{1}$ N. T. Versión libre al español hecha por la autora especialmente para este trabajo.

\section{REFERENCIAS BIBLIOGRÁFICAS}

BABICH. H.; SEGALL; M. A. \& FOX, K. D. The Allium Test - A Siimple, Eukaryote Genotoxicity Assay. The American Biology Techer, New York, v. 59, n. 9, p. 580-83. 1997.

BIEMBENGUT, M. S. Modelagem matemática no Ensino. 3. ed. São Paulo: Contexto, 2003.

BORGES, A. T. O papel do laboratório no ensino de ciências. In: ENCONTRO NACIONAL DE PESQUISA EM EDUCAÇÃO EM CIÊNCIAS, 1, 1997, Águas de Lindóia, SP. Anais. Águas de Lindóia: ENPEC, 1997.

BORGES, A. T. et.al. A resolução de problemas práticos no laboratório escolar. In: ENCONTRO NACIONAL DE PESQUISA EM EDUCAÇÃO EM CIÊNCIAS, 3, 2001, Atibaia, SP. Anais. Atibaia: ENPEC, 2001

BOTELHO, M. Projeto energia \& liberdade no atlas da diversidade: seus múltiplos significados abrindo espaços para a interdisciplinaridade. Disponível em: <http://www.isba.com.br/noticias/ artigo.doc >. Acesso em: 22/01/2006.

CARRETONI, M. Iniciacão à Matemática $1^{a}$ a $2^{a}$ série. Campinas: UNICAMP, 1986.

COTELLE, S.; MASFARAUD, J. P. \& FÉRARD, J. F. Assessment of genotoxicity of contaminated soil with the Allium: vicia-micronucleus and the Tradescantia-micronucleus assays. Mutation Research, v. 426, p. 167-171, 1999.

DA SILVA, J. \& NETO, A. S. A. DNA e Ambiente: o uso do ensaio cometa como ferramenta para discussão interdisciplinar de lesão e reparo no DNA na pós graduação em ensino de ciências. In: ENCONTRO NACIONAL DE PESQUISA EM EDUCAÇÃO EM CIÊNCIAS, 4, 2003, Bauru. Anais, Bauru: ENPEC, 2003. p. 1-13.

FISKESJÖ, G. The Allium test - a potential standard for the assessment of Environmental Toxicity and Risk Assessment. 2 vol. Philadelphia: American Society for Testing and Materials, 1993

FOUREZ, G. (2003). Crise no Ensino de Ciências?. Investigaçôes em Ensino de Ciências, Porto Alegre, v. 8 n. 2, Disponível em: <http://www.if.ufrgs.br/public/ensino/ vol8/n2/v8_n2_a1.html> Acesso em: 24/05/2006.

GALIAZZI, M. C. et al. Objetivos das atividades experimentais no ensino médio: a pesquisa coletiva como modo de formação de professores de ciências. Revista Ciência \& Educação, Bauru, v. 7 n. 2, p. 249-263, 2001.

GILBERT, J.K. \& BOULTER C. J. Developing models in science education. Dordrecht: Kluwer 2000. GOMEZ-ARROYO, S.; CASTILHO-RUIZ, P. \& VILLALOBOS-PIETRINI, R. Chromosomal alterations induced in Vicia faba by different industrial solvents: thinner, Toluene, Benzene, nHexane, n-Heptane and Ethyl Acetate. Cytologia, v. 51, p. 133-142, 1986.

HODSON, D. A critical look at practical work in school science. School Science Review. v. 70, p. 3340, 1990.

HODSON, D. (1994). Hacia un enfoque más crítico del trabajo de laboratorio. Enseñanza de las Ciências, Valência, v. 12 ,n. 3, p. 299-313, 1994.

JUSTI, R. S. \& GILBERT, J. K. A natureza de modelos na visão de professores de ciências. In: ENCONTRO NACIONAL DE PESQUISA EM EDUCAÇÃO EM CIÊNCIAS, 3, 2001, Atibaia. Anais, Atibaia: ENPEC, 2001.

KRAPAS, S.; QUEIROZ, G.; CONLINVAUX, D. \& FRANCO, C. (1997). Modelos: uma análise de sentidos na literatura de pesquisa em ensino de ciências. Investigaçôes em Ensino de Ciências. Porto Alegre, v. 2, n. 3, p. 185-205, 2007.

MAT., et al. The improvement Allium: Vicia root tip micronucleus assay for clastogenicity of environmental pollutants. Mutation Res, v. 334, p. 185-195, 1995.

MARCANO, L, et al. Cytotoxicity and mode of action of maleic hydrazide in root tips of Allium cepa. L. Environ. Res. v. 94, p. 221-226, 2004. 
NERSESSIAN, N. J. Should physicists preach what they practice? Constructive modeling in doing and learning physics. Science \& Education, v. 4, n. 3, p. 203-226, 1995.

ROGERS, C. R. Liberdade de aprender. Belo Horizonte: Interlivros, 1971.

RUTHERFORD, M. Explanations of colour. In: INTERNATIONAL HISTORY, PHILOSOPHY AND SCIENCE TEACHING CONFERENCE, 3., Minneapolis. Proceedings. Minneapolis: University of Minnesota, v. 2, p. 979-986, 1995.

SIQUEIRA, H. (2003). Formação interdisciplinar: exigência sociopolítica para um mundo em rede. VII Simpósio Estadual de Economia Doméstica. UNIOESTE. Disponível em: <http://geocities.yahoo. com.br/holgonsi/mundorede.html>. Acesso em: 22.01.2006.

SIQUEIRA, H., PEREIRA, M. Interdisciplinaridade como superação da fragmentação. Caderno de Pesquisa, Campo Grande, MS. v. 68, 1995. Disponível em: < http://geocities.yahoo.com.br/holgonsi/interdisciplinar3 >. Acesso em: 22/01/2006.

TINBERGHIN, A. Modeling as a basis for analyzing teaching-learning situations. Learning and Instruction, Oxford, v. 4, n. 1, p. 71-87, 1994.

VAN DRIEL, J. H.; VERLOOP, N. (2002). Experienced teachers' knowledge of teaching and learning of modeling in science education. International Journal of Science, London, v. 24, n. 12, p. 1255-1272.

\section{ANEXO 1: Cuestiones de pre y post-test.}

1) En el estudio de las fracciones, ¿qué entiende usted por:

a) conjunto discreto?

b) conjunto continuo?

2) ¿Qué entiende usted al observar este gráfico?

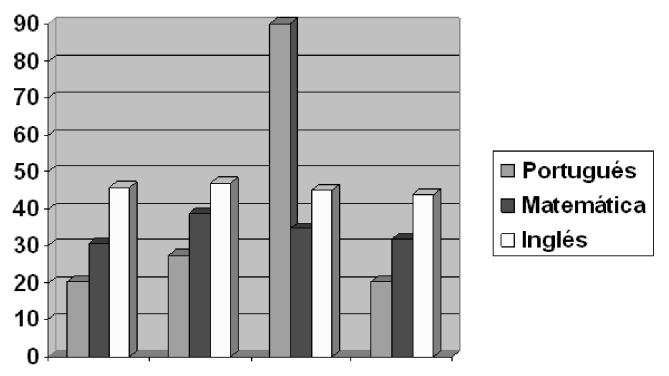

